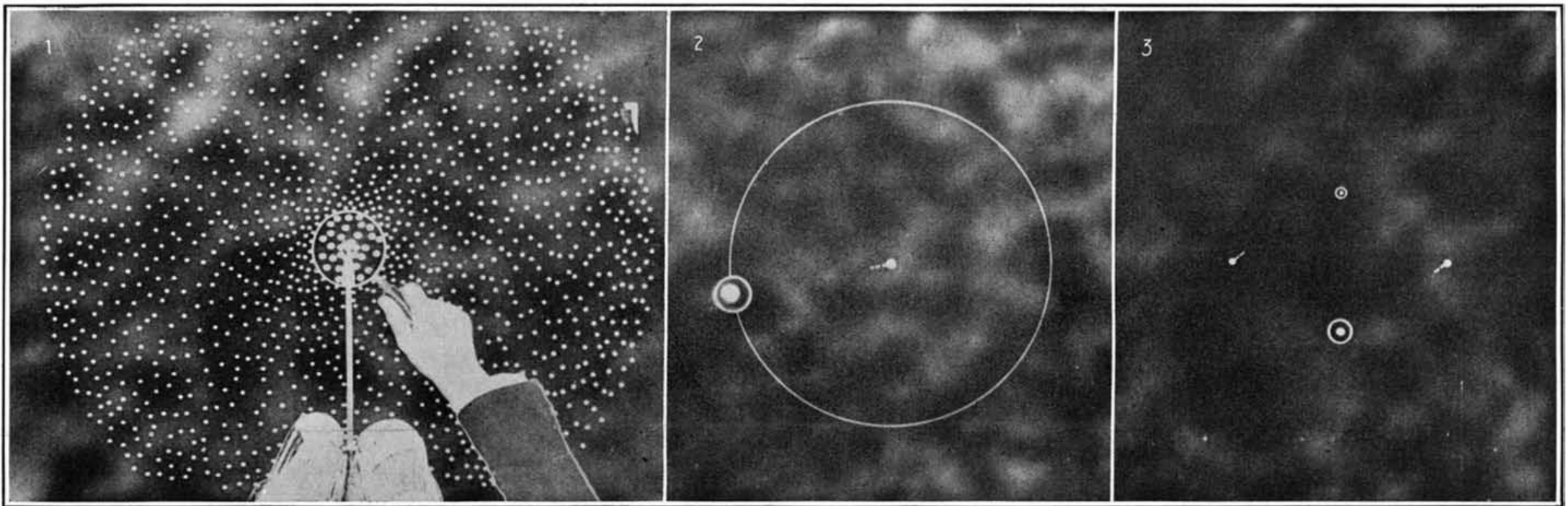

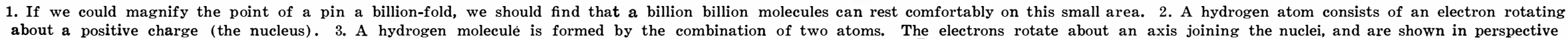

How the simplest molecule is formed, something about its size, and the structure of the atoms of which it is built

\title{
Beyond the Microscope
}

\section{A Scientific Motion Picture Film That Depicts the Structure of the Atom}

\author{
By S. Dushman, Ph.D., Research Laboratory, General Electric Co.
}

\author{
Contributing Editor, Scientific American
}

W E intend to discuss in this article some of the ing features of the new views regarding inte the discussion with still pictures taken from the film referred to in the Editor's note.

Of course, as most people know, we cannot see an atom. With even an extremely powerful microscope the smallest particles we can see are at least one thousand times larger in diameter than even the largest of molecules.

Yet we are just as certain of the existence of molecules and the atoms, of which molecules are made up, as we are of the existence in each of our bodies of certain organs, although we have never seen them. In both cases, the evidence is what is known in law as circumstantial; and, for lack of space, we shall take it for granted that all matter consists of molecules and atoms.

We know a great deal about these atoms and molecules. Thus the smallest drop of water (about 1/20 cubic centimeter) contains so many molecules that if these were each enlarged to the size of grains of sand capable of passing through a 100-mesh sieve, they would form a roadway from New York to San Francisco approximately half a mile in width and one foot in height. The molecules are so small that, as shown in Fig 1 , one billion billion could rest comfortably on the point of a pin, 1 mil in diameter.

Or perhaps the best figure is that employed by Aston in his recent popular lectures. Suppose we had means of separating out the individual molecules in a glass of water and labeling them so that we might recognize them again. Suppose then we emptied the glass into the oceans; after millions of years had elapsed to mix the water from our tumbler thoroughly and uniformly with that of the seven seas, suppose we came back and took at random a fresh glass of water from the ocean-we would find in it no less than 1,000 of our labeled molecules. That is to say, the glass of water is to the individual molecule

as the combined oceans of a thousand globes like the earth would be to the glass of water.

It is difficult to imagine that such a small particle can have a structure of its own. In fact, the word "atom" is derived from a Greek word signifying "indivisible," but while we still adhere to the term atom, we must, in the light of present knowledge, forget about the indivisibility. But we have all kinds of evidence that the atom is made up of still smaller particles which are nothing else than positive and negative electric charges. Since matter, under ordinary conditions, is electrically neutral, the numbers of positive and negative charges in any one atom are equal, but the actual number of each kind of charge differs from one kind of atom to another. The atom of hydrogen has the simplest structure of all. As shown in Fig. 2, it consists of a single negative charge, or electron, as it is designated, which rotates around the positive charge or nucleus. The electron itself is probably about a thousand times smaller in diameter than the atom. Its mass is about 2,000 times smaller than that of the atom.

Since a billion billion atoms of hydrogen more or less would hardly be noticed on the most sensitive balance this means that the mass of the electron is indeed very small.

It follows that practically the whole mass of the atom is concentrated at the nucleus. Yet this nucleus

two atoms of hydrogen combine very readily to form the hydrogen molecule, shown in Fig. 3 . The electron now rotate around an axis formed by the line joinin the two nuclei.

Hydrogen is the lightest of all elements. As we pass to the other elements, we find that the number of positive charges on the nucleus, and consequently the number of electrons around the nucleus, increase regularly. Each element can be thought of as being built up from the element just below it by the addition of one electron and one positive charge on the nucleus. Thus helium consists of two electrons and a nucleus of two unit charges; lithium has three electrons and a nucleus of three unit charges, and so forth. The heaviest atom known, that of uranium, has 92 electrons and a positive charge on the nucleus of 92 units. The most recent theory regarding the arrangement of these electrons around the nucleus in the different atoms, is that suggested by Dr. Langmuir. This theory, which is a development of some earlier speculations of Prof. G. N. Lewis of the University of Berkeley, California, postulates that the electrons are arranged in shells with the nucleus as center and that arrangements of two and eight electrons are extremely stable. In the simplest atoms, those of the hydrogen and helium, there is only one shell. With the addition of a third electron, a new shell begins to form. This shell is completed when it contains eight electrons, which may be considered as located at the corners of a cube. The atom thus obtained is that of neon, a gas which has properties quite similar to those of helium. Both are absolutely iner chemically, gases at ordinary temperatures, and very difficult to liquefy, which is in accord with the theory that a shell of eight or one of two electrons is a very stable arrangement. On the other hand, an atom containing less than eight electrons in the second shell is quite active chemically. Thus lithium, with only one electron in the

is at least 100,000 times smaller in diameter than the atom.

The electron constitutes the ultimate unit of electri charge. Electrons streaming through a conductor constitute what we measure as electric current. Prof. $R$. A. Millikan has measured accurately the charge carried by an electron; when, for instance, a 40-wat lamp is run on a 120-watt circuit, there are 2 billion billion electrons passing into the filament, per second at one lead and the same number leaving it, per second, at the other lead.

The hydrogen atom consisting of a single electron rotating around a positive nucleus of unit charge has fairly large stray field of force around it. Consequently outer shell, is extremely electro-positive, since it tends to rive up this extra electron. This leaves only the inner shell of two electrons and a nucleus of three positive units. The arrangement of electrons is thus similar to that obtained in the helium atom. The only difference is that the latter is electrically neutral, while the lithium atom which has one electron removed has a residual positive charge of one unit. It is known as a lithium ion, and is present in aqueous solutions of lithium salts, thus accounting for the electrical conductivity of such solutions.

On the other hand the fluorine atom has one electron and one positive charge less than neon. When fluorine is combined with lithium it completes the external shell 
of eight electrons by taking the extra electron from the lithium atom. Fluorine is therefore said to be electro-negative, and the fluorine ion has the same arrangement of electrons as neon, but since it has a nucleus with only seven positive charges, the resultant charge on the ion is negative. If a solution of LiF (lithium fluoride) is electrolyzed, the positively charged lithium ion goes to the cathode (negative electrode) and the negatively charged fluorine ion goes to the anode.

When one electron is added to the atom of neon, a third shell begins to form. The atom of sodium contains 11 electrons, and, like lithium, is electropositive. As more electrons are added, we obtain less strongly electropositive elements and with 7 electrons in this shell, we obtain the atom of chlorine, which resembles that of fluorine. On the addition of one more electron, we again obtain a stable, chemically inert atom, that of argon.

The fourth and fifth shells contain 18 electrons each. In each case the atom corresponding to the completed shell is that of an inert gas resembling helium, neon and argon. Thus the atom of krypton consists of five shells or layers, and has a total of $2+8+8+18+18=54$ electrons

Fig. 4 shows a model which may be used to illustrate the arrangement of electrons in any of the atoms of the first (that is lightest) 54 elements. It consists of miniature lamps, each of which is connected to a switch shown on the switchboard at the right. The nucleus is indicated by a red light at the center, and different colors on the lamps are used to bring out more sharply the different layers of electrons.

It was obviously impossible to represent It plex atoms. Moreover we do not know very much comthe actual motions of the electrons. The positions of the lamps in the model are to be taken more as indicating average locations than as fixed positions of the elecaverage locations than as fixed positions of the elec-
ronst. In order to illustrate in the film the mechanism by which compounds are formed, a simpler case was chosen, that of the combination between hydrogen and oxygen to form water. In this reaction an atom of oxygen combines with a molecule of hydrogen.

IVe have already discussed the structure of the hydrogen molecule. 'The oxygen atom is illustrated in Fig. 5. The electrons here are arranged in two shells. The inner shell has the structure of helium, that is two electrons near the nucleus. The outer shell has six electrons arranged at the corners of the imaginary cube described already. Altogether there are eight electrons and the nucleus has a positive charge of eight units. It is evident from what has been stated above, that in order to complete the outer shell, two electrons are required. These are furnished by two hydrogen atoms, that is, one molecule of hydrogen. As these electrons enter into the vacant corners of the cube, the nuclei of the hydrogen atoms tend to place themselves symmetrically with respect to two corners. Owing to the attractive forces exerted, the electrons at these corners are pulled in and the cube becomes distorted into the form shown in Fig. 6. In this figure, the nuclei of the two hydrogen atoms and that of the oxygen atoms lie on the axis shown by the dotted line, while the electrons presumably revolve about this axis and also two other axes (indicated by dotted lines) at right angles to that

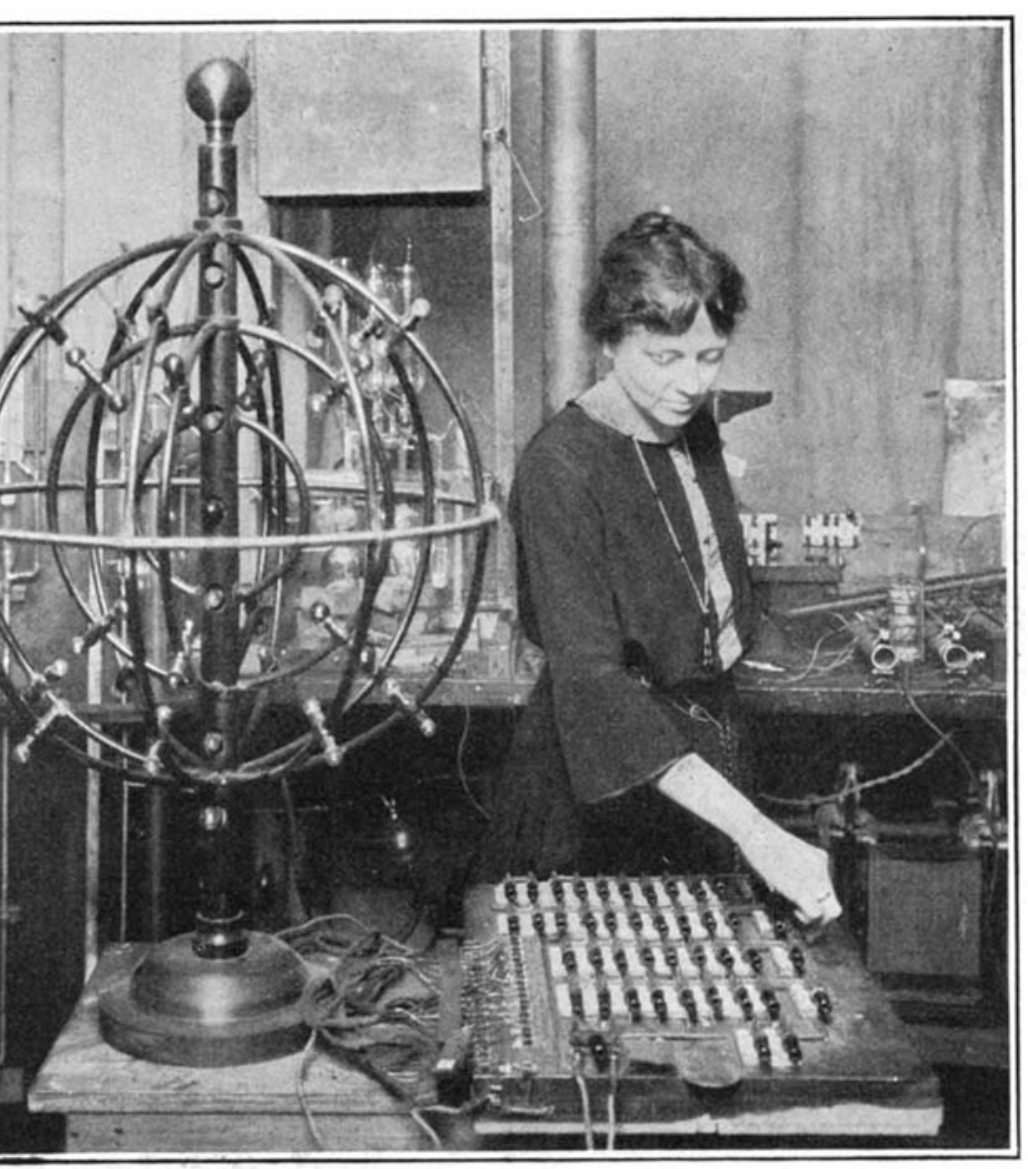

The atomic model which, by lighting any desired combination of its $\mathbf{5 4}$ Thing lights together with the central lamp representing the nucleus, ay be made to serve as a model of any of the 54 simplest atoms

Bat Guano in Austria

M UCH interest has been aroused in discovery that many of the hundreds of caverns found in the Austrian Alps contain valuable deposits of bat guano and other phosphates of animal origin. The government has passed a law retaining these under the control of the state and has been having official analyses of the deposits made and experiments as to the best method of preparing them for use as fertilizers. It has already been officially estimated that the Cave of the Dragon in Styria is capable of yielding 50,000 tons of granular phosphate of very homogeneous character: this contains 41 per cent of phosphate of lime, 25 per cent of carbonate of lime, and 34 per cent of readily attack able silicates. These fertilizers are now being systematically distributed in the different parts of Austria, to be used in replacing bone dust, etc.

New Studies of Caries in Teeth

$T$ HE alarming prevalence of caries in 1 the teeth of civilized nations is a matte of universal concern. Some new and rather surprising light is thrown upon this subject by recent investigations conducted in the laboratory of the hygienic institute of the University of Leipzig. Perfectly sound teeth taken from various individuals between the ages of 18 and 55 years were employed in the experiments. Not the slightest flaw was to be found in any of these, not even with a magnifying glass. For the purpose of the experiment each tooth was covered with a layer of wax, in which apertures were then made joining the nuclei. Of course, this particular kind of motion of the electrons is hypothetical, but it is quite probable that the electrons are not absolutely stationary. The transition from this representation of the molecule of water to the more conventional formula Fig. 7 is evident. In electrolysis, one of the hydrogen nuclei tears itself away from the molecule and as hydrogen io (with unit positive charge) travels toward the cathode while the residual part of the molecule, as negatively charged $-\mathrm{OH}$ ion, travels toward the anode.

When water is evaporated, we obtain steam. The molecules which were quite crowded together in the former case are now separated by fairly large distances and their continual impacts on the walls of the vesse produce the effect of pressure.

In water itself, the molecules are quite mobile, but when water freezes to form ice or snow, we find these molecules assume regular crystalline arrangements and the most beautiful creations in nature are those which are exhibited by snow flakes.

It is unfortunate that we cannot reproduce on the printed page the actual motion of the film. We believe that we have made as advantageous as possible a selection of our stills ; but no set of stills ever does full justice to a film. The film, made on the animated cartoon principle, gives a startlingly vivid representation of the behavior and the constitution of two of the simplest atoms. To attempt to represent in a similar manner the motions of electrons in some of the more complex atoms and the manner in which these atom combine to form compounds would, indeed, be quite task for even the motion-picture artist. at one point or another, after which the tooth was immersed in a mixture of bread and saliva, at temperature of 37 degrees Centigrade. At the end of a week half of this mixture was removed and replaced by a fresh mixture of the same sort, in order that the conditions usually present in the mouth, of a mixture of fresh food and saliva, together with decomposition products of former mixtures, might be reproduced as closely as possible. The first traces of caries became manifest in a deeply grooved incisor: softening and discoloration was shown in this tooth even after the lapse of 33 days. At the end of 56 days all the other teeth exhibited signs of softening, while completely developed caries was exhibited in all at the end of 124 days. The caries was produced at the selected points, whether these were covered with enamel or were at the "neck" of the tooth According to Professor A. Seitz, who discusses the matter in the Münchener medizinischen Wochenschrift, the caries produced in this artificial manner is precisely like natura caries, showing all the stages thereof. Strange to say, however, the common belief that marmalades and similar sweets are largely responsible for the spread of caries was not confirmed: "While mixtures of bread, saliva and marmalade produced caries in about the same length of time as the first mixture (of bread and saliva alone), teeth placed in mixtures consisting only of marmalade and saliva showed no alteration even at the end of 61 days. It appears, therefore, that both the preformed acids and the traces of natural fruit acids of the marmalade are without effect in the production of caries."-Abstracted from Die Umschau.

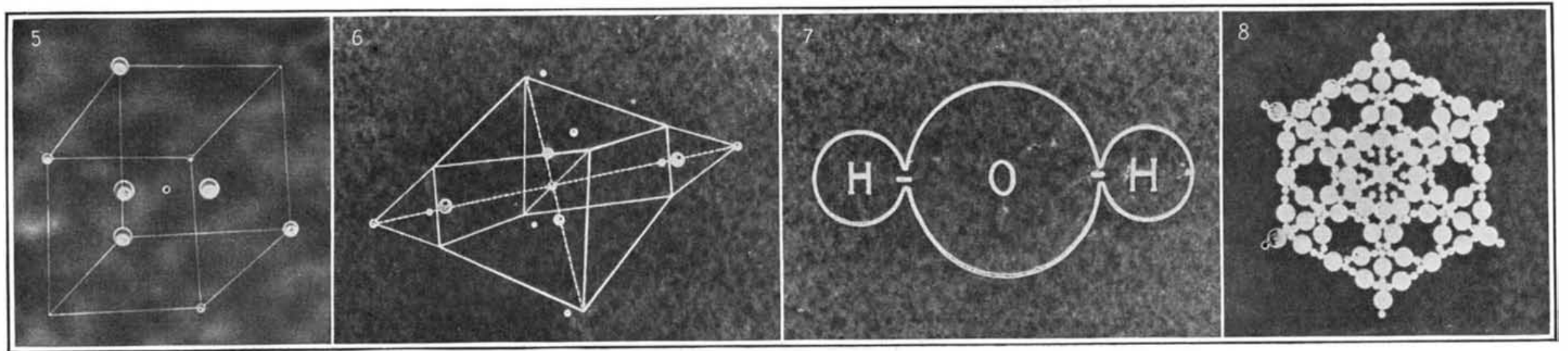

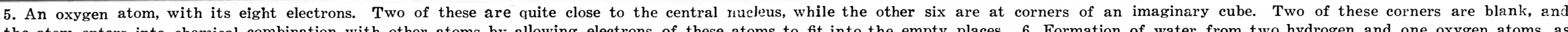

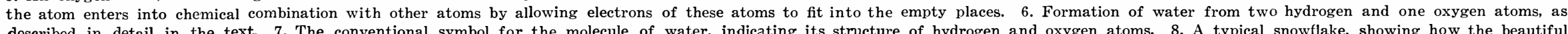

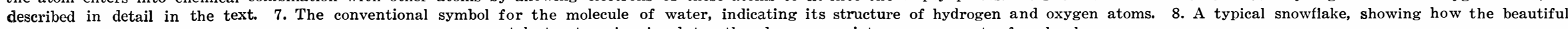

Further stills selected from the motion-picture story of the atom 